\title{
Synthesis, Leishmanicidal and anticancer activity of 4-(2-keto-1-benzimidazolinyl) piperidine and 5- chloro-1-(4-piperidyl)-2-benzimidazolinone and their derivatives
}

\author{
Tabinda Zarreen Mallick \\ Jinnah Sindh Medical University \\ Zafar Saied Saify \\ University of Karachi \\ Shazia Haider \\ University of Karachi \\ Seema Ashraf \\ University of Karachi \\ Iffat Saeed \\ University of Karachi
}

Nasreen Begum ( $\sim$ alinasreen29@yahoo.com )

University of Karachi HEJ Research Institute of Chemistry

\section{Research Article}

Keywords: Anticancer activity, benzimidazolinyl piperidine, potent, leishmanicidal activity, synthesized, Hela cells

Posted Date: February 18th, 2022

DOI: https://doi.org/10.21203/rs.3.rs-1362359/v1

License: (c) (i) This work is licensed under a Creative Commons Attribution 4.0 International License. Read Full License 


\section{Abstract}

In the current study a series of 4-(2-keto-1-benzimidazolinyl) piperidine have been newly synthesized and activities of 5-chloro-1-(4-piperidyl)-2-benzimidazolinone derivatives that is already reported have been tested and compared. Furthermore synthesized derivatives have been characterized by physical and spectral methods (HR-EIMS, HR-FABMS, ${ }^{1} \mathrm{H}-\mathrm{NMR},{ }^{13} \mathrm{C}-\mathrm{NMR}$, UV, and FT-IR). All the derivatives were tested for leishmanicidal and anticancer activities. Results demonstrated that among all the derivatives compounds 4, 9, and 10 exhibited significant cytotoxic effects on HeLa cells and therefore tested at 10, 50 and $100 \mu \mathrm{M}$ concentrations. Among all the eleven derivatives eight derivatives were found having leishmanicidal activity. Compounds $3, \mathbf{4}$ and $\mathbf{1 0}$ exhibited significant activity with $\mathrm{IC}_{50}$ values in range of $7.06 \pm 0.17 \mu \mathrm{g} / \mathrm{mL}-84.6 \pm 0.6 \mu \mathrm{g} / \mathrm{mL}$. Furthermore, an attempt was made to develop a preliminary structure-activity relationship. Compound 4 found to be most active with $\mathrm{IC}_{50}=7.06 \pm 0.17 \mu \mathrm{g} / \mathrm{mL}$ against leishmaniasis. Compounds 4 and 10 possess both leishmanicidal and anticancer activities. From this study we identified a new class of compounds having potent leishmanicidal activity and anticancer activity.

\section{Introduction}

There are many similarities exist between cancerous cells and parasitic cell. It leads to the idea that drugs use to combat parasites can be used against cancer or vice versa (Klinkert \& Heussler, 2006). Cancer remains one of the greatest challenges. It causes around $13 \%$ of all deaths worldwide and it is constantly increasing, particularly in the developed countries. Scientists have to explore either alternative mode of action to treat the disease or design newer and better drug entities to deal with the difficulties and sufferings of cancer patients (Elnima, Zubair, \& Al-Badr, 1981; Gellis, Kovacic, Boufatah, \& Vanelle, 2008). Leishmaniasis affects 12 million people around the world with an annual death rate of approximately 80,000 people. It is a widespread parasitic disease caused by protozoan parasite of the genus Leishmania. It causes a broad spectrum of clinical manifestations ranging from self-healing cutaneous lesions to the fatal visceral forms. Clinical manifestations depend on the Leishmania species involved and ranges from a life-threatening systemic infection (visceral, VL) to self-limiting or chronic skin sores (cutaneous, CL), or dreaded metastatic complications that can cause facial disfigurement (mucosal, $\mathrm{MCL}$ ). The clinical features of VL generally include prolonged and irregular fever, often associated with rigor and chills, hepato-splenomegaly, lymphadenopathy, progressive anemia, weight loss and hyper gamma globulinemia (mainly IgG from polyclonal B cell activation) and concomitant hypo-albuminemia. African and IndianVL patients may present with a secondary form called post kala-azar dermal leishmaniasis (Saha, Mukhopadhyay, \& Chatterjee, 2011). Several drugs are available for treatment of leishmaniasis, pentavalent antimonials compounds are drugs used in first line chemotherapy. As second line amphotericin B and pentamidine isothionate may be used, but current treatment is unsatisfactory due to the route of administration, unaffordable cost and toxic side-effects. That's why scientists are currently working to develop new, effective and affordable molecules as leishmanicidal agents. Fuertes et a/ reported that anticancer agents may constitute in the near future a good source of lead compounds 
against leishmaniasis and other parasitic diseases. On the other hand, it is also likely that some antiparasitic drugs may be used for the treatment of certain types of tumors (Carballeira et al., 2018; Perez, Fuertes, Nguewa, Castilla, \& Alonso, 2008; Santiago et al., 2012).

Benzimidazolinyl piperidine derivatives have recently attracted attention as an important class of heterocyclic compounds in the field of medicinal chemistry. Benzimidazolinyl piperidines have been potential target for synthesizing different derivatives having biological activities, i.e. the reason there are a number of derivatives patented by different pharmaceutical companies (Bender et al., 1996; "Benzimidazolinyl piperidines," 1965; Budzik et al., 2012; Jan \& Van, 1975; Janssen, 1965; Patel, Bhatt, Bhatt, \& Joshi). Study revealed novel benzophenone benzimidazole analogs exhibited in-vivo tumor inhibition (Croce, 2008; Ranganatha et al., 2013). Clearly, there is a need for a search for new types of drugs with high selectivity, minimum side effects and low manufacturing costs.

In the present study 11 derivatives of 4-(2-keto-1-benzimidazolinyl) piperidine and 5-chloro-1-(4piperidyl)-2-benzimidazolinone derivatives have been synthesized and tested for leishmanicidal and anticancer activity. Numbers of analogues have showed potential for cancer activity.

\section{Results And Discussion}

\subsection{Synthesis}

We report here the synthesis of 4-(2-Keto-1-benzimidazolinyl)piperidine (KBIP) derivatives $1-6$ by reaction of KBIP with a variety of acetophenones represented as $\mathrm{R}$ as shown in Table 1. We selected 2-Bromo - 4'Flouroacetophenone, 2-Bromo-4'Chloroacetophenone, 2-Bromo-2',5' dimethoxy acetophenone, (1Adamantyl bromomethyl ketone, 6-(Chloromethyl) Uracil, and 6-(Chloroacetyl)- 2H-1,4-benzoxazin$3(4 \mathrm{H})$-0ne substituents, in order to study the effect of different substituent on the (KBIP) of parent molecule. The structures of all synthesized compounds were elucidated by using different physical and spectral method (HR-EI-MS, HR-FABMS, ${ }^{1} \mathrm{H}-\mathrm{NMR},{ }^{13} \mathrm{C}-\mathrm{NMR}, \mathrm{UV}$, and FT-IR).

\subsection{Anti-Proliferation Activity in Treated HeLa Cells}

Anti-proliferation activity in treated HeLa cells has been carried out according to the literature protocol(Wang, Yang, Petrenko, \& Torchilin, 2010). Among 1-11 synthesized derivatives few compounds exhibited anti-cancer potential at a 100 uM concentration (Table-3). Concentration dependent decline in cellular viability was observed during study. The growth inhibition of HeLa cell line was observed at all concentrations after exposure to these synthesized compounds. Later the percent inhibition was calculated, which revealed greater than $50 \%$ inhibition at $100 \mu \mathrm{M}$ concentration. The $\mathrm{IC}_{50}$ values of these compounds were calculated using the ED50V10 Excel add-in. The experiment was conducted three times in duplicate. Compounds 4, 9 and 10 exhibited significant cytotoxic effect on HeLa cells and tested at 10, 50 and $100 \mu \mathrm{M}$ concentrations (Table 4-5). Cytotoxic effect of compounds 4, 9 and 10 were observed after $24 \mathrm{~h}$ treatment showing $\mathrm{IC}_{50}$ of $57.619,56.594$ and $27.235 \mu \mathrm{M}$, respectively. The error bars represent 
the SEM from the mean significantly different in treated cells compared to untreated control cells $(p<$ 0.05). The cell titer blue assay was used to assess the metabolic activities of cancer cells. The viable cells showed enhanced fluorescence whereas the cells experiencing the cytotoxic effect demonstrated a reduced fluorescence. Varying concentrations of the three potent compounds were employed on HeLa cells for $24 \mathrm{~h}$ to achieve the $\mathrm{IC}_{50}$ drug response, which represented the concentration of drug necessary to induce a reduction of $50 \%$ in growth. The compounds 4,9 and 10 exhibited a strong cytotoxic action on human cervical cancer cells, even at the lower concentrations of 10 and $50 \mu \mathrm{M}$. It can be elicited that the synthesized compounds 4, 9 and 10 demonstrated a decline in cellular functions of human cervical cancer cell as observed by a decrease in fluorescence in a concentration dependent manner (Wang et al., 2010).

\subsection{Leishmanicidal Activity}

Numbers of attempts have been made to discover potent antileishmanial drugs with less toxicity. The aim of present study is to investigate the in-vitro leishmanicidal activity of benzimidazolinyl piperidine derivatives in comparison with the standard leishmanicidal drugs amphotericin $B\left(\mathrm{IC}_{50}=0.29 \pm 0.05\right.$ $\mu \mathrm{g} / \mathrm{mL})$ and pentamidine $\left(\mathrm{IC}_{50}=5.09 \pm 0.09 \mu \mathrm{g} / \mathrm{mL}\right)$. All synthesized derivatives $1-11$ of 4 -(2-keto-1benzimidazolinyl)piperidine (I) and 5-chloro-1-(4-piperidyl)-2-benzimidazolinone (II) were evaluated for their leishmanicidal activity, (Saied Saify et al., 2014). Results showed most of them are active againstextracellular promastigotes of Leishmania major. Results are presented in table-6 along with their $\mathrm{IC}_{50}$ values. Comparing SAR of different Benzimidazolinyl Piperidine derivatives, it can be concluded that the slight changes in structure, functional groups and their position play important role in making compound active or inactive. Among 1-11 synthesized derivatives eight compounds were found active. Compounds 3,4 and 10 exhibited significant activity with $\mathrm{IC}_{50}$ values in range of $(7.06 \pm 0.17 \mu \mathrm{g} / \mathrm{mL}$ to $84.6 \pm 0.6 \mu \mathrm{g} / \mathrm{mL}$ ), Compounds 1 and 7 showed good activities while 2, 8 and 9 exhibited low activity. Furthermore, an attempt was made to develop a preliminary structure-activity relationship for the synthetic compounds. Compound 4 found to be most active with $\mathrm{IC}_{50}=7.06 \pm 0.17 \mu \mathrm{g} / \mathrm{mL}$ against leishmaniasis. Compound 10 also showed significant result with $\mathrm{IC}_{50}=23.24 \pm 0.035 \mu \mathrm{g} / \mathrm{mL}$ there is a 3 times reduction in leishmanicidal activity in compound $\mathbf{1 0}$ due to the chloro group substituent present at benzimidazolinone ring. Both the derivatives possesses adamantane ring indicated that it is responsible for significant leishmanicidal activity. Compound $\mathbf{3}$ also showed significant activity possibly due to the presence of two electron donating methoxy group substituents present at ortho and meta positions of phenyl ring. Compound $\mathbf{1}$ and $\mathbf{7}$ also showed good activity. These compounds are phenacyl halide derivative of benzimidazolinyl piperidine. They have fluorine group at para position of benzene ring both possesses comparable leishmanicidal activity. Compounds $\mathbf{8}$ and $\mathbf{9}$ showed weak activity. On the other hand Compound 5, 6, 11 and parent I showed devoid of activity against leishmaniasis. Parent molecule I found inactive while interestingly II exhibited moderate activity. Generally all newly synthesized derivatives showed promising activity. These investigations suggested that compounds $\mathbf{4}$ and $\mathbf{1 0}$ may serve as lead compound in search of anti-leishmanial drugs. It has been reported that anticancer agents may constitute in the near future a good source of lead compounds against leishmaniasis and other 
parasitic diseases(Perez et al., 2008). On the other hand, it is also likely that some antiparasitic drugs may be used for the treatment of certain types of tumors. Due to their drug-like properties, this series of compounds can potentially serve as templates for future drug-optimization and drug-development efforts for use as therapeutic agents.

\section{Experimental}

\subsection{General Experimental Conditions}

All reagents used were purchased from Sigma Aldrich Company and organic solventswere of analytical grade. Watt man's filter paper was used for filtration. Thin layer chromatography (TLC) was performed on pre-coated silica gel aluminum plates (Kieselgel 60, 254, E. Merck, Germany). Chromatograms were visualized by UV at 254 and $365 \mathrm{~nm}$. The synthesized derivatives were dried and stored in vacuum anhydrous condition. Melting points of products were noted on Buchi 434 melting point apparatus and are uncorrected. Hitachi U-3200 spectrophotometer used to record Ultraviolet (UV) spectra while methanol used as solvent. Infra-Red (IR) spectra were analyzed on a Jasco 302 Fourier transform FTIR spectrophotometer by making KBR disc method. Mass spectrometry were carried out on Varian Massen spectrometer MAT 311A spectrometer Varian Massen spectrometer MAT 312, MAT 113 DMASPEC system. Nuclear magnetic resonance ${ }^{1} \mathrm{HNMR}$ and ${ }^{13} \mathrm{CNMR}$ spectral analysis were carried out at Bruker AM 300, 400 and 500 MHz spectrophotometers. Solvents used were DMSO- $d_{6}$ and MeOD. Chemical shifts $(\delta)$ were recorded in parts per million (ppm) and coupling constants $J$ in Hertz.

\subsection{General procedure for Synthesis of 4-(2-Keto-1- benzimidazolinyl) piperidine1-6 Derivatives}

4-(2-Keto-1-benzimidazolinyl)piperidine derivatives 1-6 have been synthesized by refluxing equimolar 4(2-Keto-1-benzimidazolinyl)piperidine (I) with variety of phenacyl halides groups $(\mathbf{R})$ dissolved in methanol in a round bottom flask. Synthesis of 5-Chloro-1-(4-piperidyl)-2-benzimidazolinone derivatives 7-11 have been reported previously by (HAIDER, SAEED, SULTANA, \& KHAN, 2014). The reaction mixture was refluxed vigorously by magnetic stirring for $2 \mathrm{~h}$ at $25^{\circ} \mathrm{C}$. The progress of reaction was monitored by TLC technique. The crude solid products were filtered and washed with acetone. The crude products were recrystallized using warm ethyl alcohol and diethyl ether. The pure product was dried in desiccator using anhydrous calcium sulphate.

\subsection{1 [1-(2-(4-fluorophenyl)-2-oxoethyl)-4-(2-oxo-2, 3- dihydro-1H-benzo[d]imidazole-1-yl)piperidinium bromide] (1)}

Yield $80.5 \%$, White crystals, mp: $250.7 \pm 1.1^{\circ} \mathrm{C}, \mathrm{C}_{20} \mathrm{H}_{21} \mathrm{O}_{2} \mathrm{~N}_{3} \mathrm{~F}$ (354.1540); Solubility (MeOH, EtOH, DMSO); $\mathrm{UV}_{\lambda \max }(\mathrm{MeOH})(\mathrm{nm}): 207,232,249,281 ; \mathrm{FT}-\mathrm{IR} \mathrm{U}_{\max }(\mathrm{KBr}) \mathrm{cm}^{-1}:$ 700, 727, 1104, 1198, 1387, 1486, 1624, 
2945, 3155, 3448, 3190; HR-FABMS: $354.1629\left\{\left[\mathrm{M}+\mathrm{H}^{+} \mathrm{C}_{20} \mathrm{H}_{21} \mathrm{O}_{2} \mathrm{~N}_{3} \mathrm{~F}\right\}\right.$ (Calcd 354.1540); ${ }^{1} \mathrm{H}$-NMR: $\left(d_{6^{-}}\right.$ DMSO, $400 \mathrm{MHz}) \delta \mathrm{H}(\mathrm{ppm}): 1.93(\mathrm{~d}, 2 \mathrm{H}, J=12.0 \mathrm{~Hz}), 2.81(\mathrm{~d}, 2 \mathrm{H}, J=12.0 \mathrm{~Hz}), 2.81(\mathrm{~d}, J=12.0 \mathrm{~Hz}, 2 \mathrm{H})$, $3.64(\mathrm{~d}, J=8.0 \mathrm{~Hz}, 4 \mathrm{H}), 4.58$ (br. s, 1H), 5.07 (s, 2H), 7.02, (m, 3H), $7.47(\mathrm{~m}, 3 \mathrm{H}), 8.10(\mathrm{~m}, 2 \mathrm{H}), 10.9(\mathrm{~s}, 1 \mathrm{H})$; ${ }^{13} \mathrm{C}$-NMR $\left(d_{6}\right.$-DMSO, $\left.100 \mathrm{MHz}\right) \delta \mathrm{C}(\mathrm{ppm}): 25.5$ (C-3, 5), 46.4 (C-4), 52.9 (C-2, 6), 61.2 (C-7), 108.7 (C-18), 109.1 (C-15), 116.1 (C-21), 116.4 (C-23), 120.9 (C-16), 120.3 (C-17), 128.3 (C-13), 128.5 (C-14), 130.5 (C19), 131.3 (C-20), 131.4 (C-24), 153.5 (C-11), 167.1 (C-22), 190.2 (C-8).

\subsection{2 [1-[2-(4-Chloro-phenyl)-2-oxo-ethyl]-4-(2-oxo-2, 3- dihydro-benzoimidazol-1-yl)piperidinium bromide] (2)}

Yield $90.5 \%$, White shiny crystals, mp $204 \pm 0.7, \mathrm{C}_{20} \mathrm{H}_{20} \mathrm{O}_{2} \mathrm{~N}_{3} \mathrm{Cl}$ (369.12); Solubility ( $\mathrm{MeOH}, \mathrm{H}_{2} \mathrm{O}$, DMSO); $U_{V_{\lambda \max }}(\mathrm{MeOH})(\mathrm{nm}): 206.4,251.2,279.6 ; \mathrm{FT}-\mathrm{IR} \boldsymbol{U}_{\max }(\mathrm{KBr}) \mathrm{cm}^{-1}:$ 700.5, 729.1, 821.3, 932.5, 1272, 1387.5, 1485.2, 1596.4, 1624.9, 1680, 2510.5, 2638.7, 2723.2, 2816.8, 2943.7, 3071.0, 3155.6, 3190.8, 3449.5, 3660.9; HR-EIMS m/z. $369\left(\mathrm{C}_{20} \mathrm{H}_{20} \mathrm{O}_{2} \mathrm{~N}_{3} \mathrm{Cl}\right.$ Calcd 369.12); ${ }^{1} \mathrm{H}$-NMR (MeOD, $300 \mathrm{MHz}$,) $\delta \mathrm{H}$ (ppm): 2.11 (d, $J=12.0 \mathrm{~Hz}, 2 \mathrm{H}), 4.61$ (br. s, 1H), $5.00(\mathrm{~s}, 2 \mathrm{H}), 7.10(\mathrm{~m}, 3 \mathrm{H}), 7.33(\mathrm{~m}, 1 \mathrm{H}), 7.62(\mathrm{~d}, J=12.0 \mathrm{~Hz}, 2 \mathrm{H}), 8.05(\mathrm{~d}$, $J=6.0 \mathrm{~Hz}, 2 \mathrm{H}), 10.9(\mathrm{~s}, 1 \mathrm{H}) ;{ }^{13} \mathrm{C}-\mathrm{NMR}\left(100 \mathrm{MHz}, \mathrm{DMSO}-d_{6}\right) \delta \mathrm{C}(\mathrm{ppm}): 25.5(\mathrm{C} 3,5), 46.4(\mathrm{C}-4), 52.5$ (C-2, 6), 61.2 (C-7), 108.8 (C-18), 109.1 (C-15), 120.3 (C-17), 120.9 (C-16), 128.3 (C-14), 128.7 (C-13), 129.2 (C-21, 23), 130.1 (C20, 24), 132.4 (C-19), 139.7 (C-22), 153.5 (C-11), 190.7 (C-8).

\subsection{3 [1-(2-(2, 5-dimethoxyphenyl)-2-oxoethyl)-4-(2-oxo-2, 3- dihydro-1H-benzo[d]imidazole-1-yl)piperidinium bromide] (3)}

Yield $80.5 \%$, Greenish white powder, mp $177.15 \pm 1.55^{\circ} \mathrm{C}, \mathrm{C}_{22} \mathrm{H}_{26} \mathrm{~N}_{3} \mathrm{O}_{4}$ (395.18); Solubility $\left(\mathrm{MeOH}, \mathrm{H}_{2} \mathrm{O}\right.$, DMSO); $U_{\lambda_{\text {max }}}(\mathrm{MeOH})(\mathrm{nm}): 206.4,253.8,279.8 ; \mathrm{FT}-\mathrm{IR} \mathbf{U}_{\max }(\mathrm{KBr}) \mathrm{cm}^{-1}: 696,727.2,754.8,966.9,1026.3$, 1168.6, 1222.7, 1280, 1309.4, 1389.7, 1497, 1583.6, 2679.7, 2831.9, 2954.9, 3183.2, 3432.7; HR-FABMS: $396.1029\left\{[\mathrm{M}+\mathrm{H}]^{+}\left(\mathrm{C}_{22} \mathrm{H}_{26} \mathrm{~N}_{3} \mathrm{O}_{4}\right\}\right.$ (Calcd 395.18); ${ }^{1} \mathrm{H}-\mathrm{NMR}$ (MeOD, $300 \mathrm{MHz}$ ) $\delta \mathrm{H}$ (ppm): 1.90 (d, $J=12.0$ $\mathrm{Hz}, 2 \mathrm{H}), 2.79(\mathrm{~d}, J=12.0 \mathrm{~Hz}, 2 \mathrm{H}), 3.62(\mathrm{~s}, 3 \mathrm{H}), 3.77(\mathrm{~s}, 3 \mathrm{H}), 4.54$ (br. s, 1H), $4.80(\mathrm{~s}, 2 \mathrm{H}), 7.00(\mathrm{~m}, 3 \mathrm{H}), 7.25$ $(\mathrm{d}, J=8.0 \mathrm{~Hz}, 1 \mathrm{H}), 7.46(\mathrm{~d}, J=8.0 \mathrm{~Hz}, 1 \mathrm{H}), 7.34(2 \mathrm{H}, \mathrm{m}), 9.89(\mathrm{~s}, 1 \mathrm{H}) ;{ }^{13} \mathrm{C}-\mathrm{NMR}\left(100 \mathrm{MHz}\right.$, DMSO-d $\left.\mathrm{d}_{6}\right) \delta \mathrm{C}$ (ppm): 25.4 (C-3, 5), 46.5 (C-4), 52.5 (C-2), 55.7 (C-26), 56.5 (C-27). 65.0 (C-7), 113.3 (C-24), 114.5 (C-21), 122.6 (C-22), 128.3 (C-19), 128.5 (C-13), 153.0 (C-11), 194.5 (C-8).

\subsection{4 [1-(2-Adamantan-1-yl-2-oxoethyl)-4-(2-oxo-2,3-dihydro- benzoimidazol-1-yl)piperidinium bromide] (4)}

Yield 90\%, White crystals, mp $295 \pm 1^{\circ} \mathrm{C}, \mathrm{C}_{24} \mathrm{H}_{31} \mathrm{O}_{2} \mathrm{~N}_{3}$ (393.24); Solubility (MeOH, $\left.\mathrm{H}_{2} \mathrm{O}, \mathrm{DMSO}\right) ; \mathrm{UV}_{\lambda \max }$ $(\mathrm{MeOH})(\mathrm{nm}): 213,229,282 ; \mathrm{FT}-\mathrm{IR} \boldsymbol{u}_{\max }(\mathrm{KBr}) \mathrm{cm}^{-1}:$ 882.0, 958.1, 993.7, 1097.9, 1210.9, 1388.7, 1483.0, 1690.4, 2509, 2666.4, 2748.3, 2852.9, 2913.9, 3063.3, 3191.0, 3409.5; HR-EIMS m/z. $393\left(\mathrm{C}_{24} \mathrm{H}_{31} \mathrm{O}_{2} \mathrm{~N}_{3}\right.$ Calcd 393.24); ${ }^{1} \mathrm{H}-\mathrm{NMR}\left(500 \mathrm{MHz}, \mathrm{DMSO}-d_{6}\right) \delta \mathrm{H}$ (ppm): 1.67 (q, $\left.J=12.5,36.5 \mathrm{~Hz}, 6 \mathrm{H}\right), 1.79$ (d, $J=2.0 \mathrm{~Hz}$, $6 \mathrm{H}), 1.87(\mathrm{~d}, J=10.0 \mathrm{~Hz}, 4 \mathrm{H}), 2.0(\mathrm{~s}, 3 \mathrm{H}), 2.70(2 \mathrm{H}, \mathrm{m}), 3.48(2 \mathrm{H}, \mathrm{m}), 4.28(\mathrm{~s}, 1 \mathrm{H}), 3.17(\mathrm{~m}, 2 \mathrm{H}), 4.48(\mathrm{~s}, 2 \mathrm{H})$, 
$7.02(\mathrm{~m}, 3 \mathrm{H}), 7.40\left(\mathrm{~d}, J=10.0 \mathrm{~Hz}, 1 \mathrm{H}\right.$ ), $10.9(\mathrm{~s}, 1 \mathrm{H}) ;{ }^{13} \mathrm{C}-\mathrm{NMR}\left(100 \mathrm{MHz}, \mathrm{DMSO}-d_{6}\right) \delta \mathrm{C}(\mathrm{ppm}): 25.3(\mathrm{C}-3,5)$, 27.2 (C-21, 23, 26), 35.7 (C-22, 27, 28), 37.1 (C-20, 24, 25), 44.4 (C-19), 46.4 (C-4), 52.3 (C-2, 6), 59.6 (C-7), 108.7 (C-17), 109.0 (C-15), 120.2 (C-16), 120.8 (C-18), 128.3 (C-13, 14), 153.4 (C-11), 207.4 (C-8).

\subsection{5 [1-(2, 6-dioxo-1, 2, 3, 6-tetrahydropyrimidin-4-yl) methyl-4-(2-oxo-2,3-dihydro-1H-benzo[d]imidazole-1- yl)piperidinium chloride] (5)}

Yield $80 \%$, White shiny crystals, mp $189.65 \pm 1.15^{\circ} \mathrm{C}, \mathrm{C}_{17} \mathrm{H}_{19} \mathrm{O}_{3} \mathrm{~N}_{5}$ (341.15); Solubility ( $\mathrm{MeOH}, \mathrm{H}_{2} \mathrm{O}$, DMSO); $U_{\lambda} V_{\max }(\mathrm{MeOH})(\mathrm{nm}): 206.8,265.4 ; \mathrm{FT}^{-I R} \mathbf{u}_{\max }(\mathrm{KBr}) \mathrm{cm}^{-1}:$ 737.9, 837.7, 882.0, 969.2, 1006.7, 1094.6, 1147.7, 1319.1, 1380.5, 1482.7, 1690.6, 1710.3, 2819.7, 2946, 3119.2, 3434.2; HR-EIMS m/z. 341 $\left(\mathrm{C}_{17} \mathrm{H}_{19} \mathrm{O}_{3} \mathrm{~N}_{5}\right.$ Calcd 341.15); ${ }^{1} \mathrm{H}-\mathrm{NMR}\left(300 \mathrm{MHz}, \mathrm{DMSO}-d_{6}\right) \delta \mathrm{H}(\mathrm{ppm}): 1.62(\mathrm{~d}, J=9.4 \mathrm{~Hz}, 2 \mathrm{H}), 2.18(\mathrm{~m}$, 2H), $2.33(\mathrm{~m}, 2 \mathrm{H}), 2.93(\mathrm{~d}, J=10.5 \mathrm{~Hz}, 2 \mathrm{H}), 3.23(\mathrm{~s}, 2 \mathrm{H}), 4.13(\mathrm{~s}, 1 \mathrm{H}), 5.52(\mathrm{~s}, 1 \mathrm{H}), 7.01(\mathrm{~m}, 2 \mathrm{H}), 7.27(\mathrm{~d}, J=$

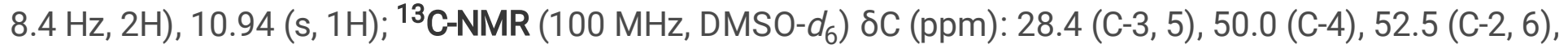
98.3 (C-18), 108.6 (C-13),109.8 (C-16),120.0 (C-14), 124.7 (C-15), 128.1 (C-12), 129.5 (C-11), 151.5 (C-9), 153.3 (C-21), 153.6 (C-7, C-17), 164.1 (C-19).

\subsection{6 [4-(2-oxo-2, 3-dihydro-1H-benzo[d]imidazole-1-yl)-1-(2- oxo-2-(3-oxo-3, 4-dihydro-2H-benzo[b][1,4]oxazin-6-yl)ethyl) piperidinium chloride] (6)}

Yield $92 \%$, White fluffy powder, mp $251 \pm 1^{\circ} \mathrm{C}, \mathrm{C}_{22} \mathrm{H}_{23} \mathrm{~N}_{4} \mathrm{O}_{4}$ (406.16); Solubility ( $\mathrm{MeOH}, \mathrm{H}_{2} \mathrm{O}$, DMSO); $U_{V_{\lambda \max }}(\mathrm{MeOH})(\mathrm{nm}): 207.8,243.4,279.6 ; F^{\prime}-I R U_{\max }(\mathrm{KBr}) \mathrm{cm}^{-1}:$ 927.3, 1039.6, 1095.4,1159.6, 1282.0, 1385.5,1417.1, 1499.5, 1696.8, 2771.6, 2858.2, 2954.1, 3053.1, 3213.1, 3698.4; HR-ESI m/z. 407.16 $\left(\mathrm{C}_{22} \mathrm{H}_{23} \mathrm{~N}_{4} \mathrm{O}_{4}\right.$ Calcd 406.16); ${ }^{1} \mathrm{H}-\mathrm{NMR}\left(300 \mathrm{MHz}\right.$, DMSO- $\left.d_{6}\right) \delta \mathrm{H}(\mathrm{ppm}): 1.62(\mathrm{~d}, J=11.4 \mathrm{~Hz}, 2 \mathrm{H}), 2.26(\mathrm{~m}$, 2H), $2.31(\mathrm{~m}, 2 \mathrm{H}), 2.60(\mathrm{~m}, 2 \mathrm{H}), 3.0(\mathrm{~d}, J=9.3 \mathrm{~Hz}, 1 \mathrm{H}), 3.77(\mathrm{~s}, 2 \mathrm{H}), 4.68(\mathrm{~s}, 1 \mathrm{H}), 6.97(\mathrm{~m}, 2 \mathrm{H}), 7.04(\mathrm{~d}, J=$ $8.4 \mathrm{~Hz}, 1 \mathrm{H}), 7.18(\mathrm{~d}, J=4.5 \mathrm{~Hz}, 1 \mathrm{H}), 7.57(\mathrm{~d}, J=2.1 \mathrm{~Hz}, 1 \mathrm{H}), 7.68(\mathrm{q}, J=2.1,8.4 \mathrm{~Hz}, 2 \mathrm{H}), 10.8(\mathrm{~s}, 1 \mathrm{H}), 10.88$ $(\mathrm{s}, 1 \mathrm{H}) ;{ }^{13} \mathrm{C}-\mathrm{NMR}$ (100 MHz, DMSO- $\left.d_{6}\right) \delta \mathrm{C}(\mathrm{ppm}): 28.5$ (C-3, 5), 52.7 (C-2, 6), 59.0 (C-4), 66.7 (C-7, C-21), 108.5 (C-19), 115.9 (C-16), 116.0 (C-26), 120.3 (C-27),120.4 (C-29),124.3 (C-18), 124.7 (C-17), 127.1 (C-24), 128.2 (C-14), 129.1 (C-28), 130.2 (C-15),147.2 (C-11), 153.6 (C-25), 164.1 (C-22), 195.3 (C-8).

\subsection{Biological Activity Evaluation}

\subsubsection{Cytotoxicity Assay}

\subsubsection{Protocol for Cervical (HeLa) Cancer Cell Line}

HeLa cells were cultured in $75 \mathrm{~cm}^{2}$ polystyrene culture flasks with canted neck and filtered caps (Corning Inc, NY, USA) in DMEM supplemented with $10 \%$ FBS and $1 \%$ (penstrep) agent. Cells were consistently provided a warm and humidified environment of $37^{\circ} \mathrm{C}$ and $5 \% \mathrm{CO}_{2}$. Cells were grown to $80 \%$ confluence 
before every experiment. The cancer cells were washed with PBS and were harvested with a solution of trypsin-EDTA whilst in a logarithmic phase of growth.

The cell plating was performed in a 96-well plate, when cells were $80 \%$ confluent. After $24 \mathrm{~h}$ the drug treatment was performed. Next day cells were exposed to the fluorescent dye, celltiter blue (CTB), the indicator compound in the CTB is blue colored resazurin, which is oxidized by the viable cells into red colored resorufin compound. Following 2 and 4 hour of incubation at $37^{\circ} \mathrm{C}$, the fluorescence is calculated in a spectro-fluorometer at $544 / 590 \mathrm{~nm}$ excitation and emission wavelength of a filter respectively (Wang et al., 2010). All experiments were repeated as a minimum of three times. Data from the experiments was assembled and the statistical significance between control and treated groups was evaluated by Student's t-test. The significance level ( $p$-value) of $p<0.05$ was considered significant.

\subsubsection{In-Vitro Leishmanicidal Assay}

For this assay Leishmania promastigotes were harvested in an excess quantity. Modified NNN biphase and RPMI 1640 mediums were used (Sigma, St. Louis, USA) supplemented with 10\% heat resistant fetal calf serum (PAA Laboratories $\mathrm{GmbH}$, Austria). Parasites on log phase of growth were centrifuged at 2000 rpm for 10 minutes. They were diluted and adjusted the cell density up to $1 \times 10^{6}$ cells $/ \mathrm{mL}$. Experiment was done in 96-well round bottom micro titer plate contains $20 \mu \mathrm{L}$ of the synthesized compound along with medium were added and serially diluted, $100 \mu \mathrm{L}$ of parasite culture was added in each well. Two rows of 96-well plates fixed for positive and negative control. Negative control wells comprises of medium whereas the positive control wells consist of varying concentrations of standard anti-leishmanial drugs. All samples were use as $1 \mathrm{mg} / \mathrm{mL}$. After that 96 well plates incubated at $22-25^{\circ} \mathrm{C}$ for 72 hours. The culture was analyzed microscopically by using Neubaure counting chamber and $\mathrm{IC}_{50}$ values of antileishmanial activity were calculated through Software Ezfit 5.03 Perella Scientific. Experiment performed in triplicate (Choudhary, Yousaf, Ahmed, \& Yasmeen, 2005; Habtemariam, 2003).

\section{Conclusions}

We have synthesized two series of 1-11 derivatives of benzimidazolinyl piperidine by taking two parent molecules one is 4-(2-keto-1-benzimidazolinyl)piperidine (I) (KBIP) and another is 5-chloro-1-(4piperidyl)-2-benzimidazolinone (II) (CPB). Derivatives $\mathbf{1 - 6}$ belong to KBIP and $\mathbf{7 - 1 1}$ belongs to $\mathrm{CPB}$. KBIP series synthesis is reporting for the first time while CPB synthesis has been reported by our research group previously. We selected these phenacyl halides to synthesize the derivatives, 2-Bromo - 4'Flouroacetophenone, 2-Bromo-4'Chloroacetophenone, 2-Bromo-2',5' dimethoxy acetophenone, (1Adamantyl bromomethyl ketone, 6-(Chloromethyl) Uracil, and 6-(Chloroacetyl)- 2H-1,4-benzoxazin$3(4 \mathrm{H})-0$ ne. Derivatives have been synthesized following a single step quarternization reaction. The synthesized compounds were characterized by using different techniques such as FT-IR, UV, ${ }^{1} \mathrm{H}-\mathrm{NMR},{ }^{13} \mathrm{C}$ NMR and mass spectrometry.

In vivo biological potential of eleven derivatives 1-11 were evaluated by using Cervical (HeLa) Cancer cell line. Compounds 4, 9, and 10 exhibited significant cytotoxic effect on HeLa cell line. They exhibited significant cytotoxic action on human cervical cancer cells, even at the lower concentrations of 10 and 50 
$\mu \mathrm{M}$. It can be elicited that the synthesized compounds $\mathbf{4 , 9}$, and $\mathbf{1 0}$ demonstrate a decline in cellular functions of human cervical cancer cell as observed by a decrease in fluorescence in a concentration dependent manner. Due to their promising results, they can be selected for further studies as lead molecule.

In vitro Leishmanicidal potentialof benzimidazolinyl piperidine derivatives 1-11 was evaluated using modified NNN biphase and RPMI 1640 mediums. Most of the synthesized compounds showed leishmanicidal potential. Compounds 3, 4, and 10 exhibited significant Leishmanicidal activities. These investigations suggest that compounds $\mathbf{4}$ and $\mathbf{1 0}$ may serve as lead compound in search of better leishmanicidal drugs.

\section{Declarations}

\section{Acknowledgment:}

Authors acknowledged ICCBS international centre for chemical and biological Sciences, for providing the research facilities to carry out this research work.

\section{References}

1. Bender, W., Hansen, J., Paessens, A., Wolfgang, R., Raddatz, S., \& Wild, H. (1996). Substituted (2-oxo1-benzimidazolinyl)-peperidines, process for their preparation, and use as anti-retroviral agents: Google Patents.

2. Benzimidazolinyl piperidines. (1965): Google Patents.

3. Budzik, B., Cooper, D. G., Forbes, I. T., Garzya, V., Jin, J., Shi, D., .. Walker, G. (2012). Compounds which have activity at $\mathrm{M} 1$ receptor and their uses in medicine: Google Patents.

4. Carballeira, N. M., Morales-Guzman, C., Alvarez-Benedicto, E., Torres-Martinez, Z., Delgado, Y., Griebenow, K. H., ... Carbajo-Andres, R. (2018). First total synthesis of $\omega$-phenyl $\Delta 6$ fatty acids and their leishmanicidal and anticancer properties. Current topics in medicinal chemistry, 18(5), 418-427.

5. Choudhary, M. I., Yousaf, S., Ahmed, S., \& Yasmeen, K. (2005). Antileishmanial physalins from Physalis minima. Chemistry \& biodiversity, 2(9), 1164-1173.

6. Croce, C. M. (2008). Oncogenes and cancer. N Engl J Med, 358(5), 502-511. doi: 10.1056/NEJMra072367

7. Elnima, E. I., Zubair, M. U., \& Al-Badr, A. A. (1981). Antibacterial and antifungal activities of benzimidazole and benzoxazole derivatives. Antimicrobial agents and chemotherapy, 19(1), 29-32.

8. Gellis, A., Kovacic, H., Boufatah, N., \& Vanelle, P. (2008). Synthesis and cytotoxicity evaluation of some benzimidazole-4,7-diones as bioreductive anticancer agents. Eur J Med Chem, 43(9), 18581864. doi: http://dx.doi.org/10.1016/j.ejmech.2007.11.020 
9. Habtemariam, S. (2003). In vitro antileishmanial effects of antibacterial diterpenes from two Ethiopian Premna species: P. schimperi and P. oligotricha. BMC pharmacology, 3(1), 6.

10. HAIDER, S., SAEED, S. M. G., SULTANA, N., \& KHAN, A. (2014). INTERNATIONAL JOURNAL OF CURRENT RESEARCH IN CHEMISTRY AND PHARMACEUTICAL SCIENCES. Int. J. Curr. Res. Chem. Pharma. Sci, 1(9), 191-202.

11. Jan, P. A., \& Van, I. (1975). 1-\{8 1-(2-Hydroxy-3-aryloxypropyl)-4-piperidyl \{9-2-benzimidazolinones and related compounds: Google Patents.

12. Janssen, R. A. J. (1965). Benzimidazolinyl piperidine derivatives: Google Patents.

13. Klinkert, M., \& Heussler, V. (2006). The use of anticancer drugs in antiparasitic chemotherapy. Mini reviews in medicinal chemistry, 6(2), 131-143.

14. Patel, V., Bhatt, N., Bhatt, P., \& Joshi, H. Synthesis and pharmacological evaluation of novel 1(piperidin-4-yl)-1H-benzo [d] imidazol-2 (3H)-one derivatives as potential antimicrobial agents. Medicinal Chemistry Research, 1-7.

15. Perez, J. M., Fuertes, M. A., Nguewa, P. A., Castilla, J., \& Alonso, C. (2008). Anticancer compounds as leishmanicidal drugs: challenges in chemotherapy and future perspectives. Current medicinal chemistry, 15(5), 433-439.

16. Ranganatha, V. L., Vijay Avin, B. R., Thirusangu, P., Prashanth, T., Prabhakar, B. T., \& Khanum, S. A. (2013). Synthesis, angiopreventive activity, and in vivo tumor inhibition of novel benzophenonebenzimidazole analogs. Life Sci, 93(23), 904-911. doi: 10.1016/j.Ifs.2013.10.001

17. Saha, P., Mukhopadhyay, D., \& Chatterjee, M. (2011). Immunomodulation by chemotherapeutic agents against Leishmaniasis. International Immunopharmacology, 11(11), 1668-1679. doi: http://dx.doi.org/10.1016/j.intimp.2011.08.002

18. Saied Saify, Z., Zarreen Mallick, T., Begum, N., Yousuf, S., Saeed, G., Muhammad, S., . . Qaiser, S. (2014). Synthesis, X-Ray Crystallography and Leishmanicidal Activity of Benzimidazolinyl Piperidine derivative. Journal of the Chemical Society of Pakistan, 36(6).

19. Santiago, I. F., Alves, T., Rabello, A., Sales Junior, P. A., Romanha, A. J., Zani, C. L., . . Rosa, L. H. (2012). Leishmanicidal and antitumoral activities of endophytic fungi associated with the Antarctic angiosperms Deschampsia antarctica Desv. and Colobanthus quitensis (Kunth) Bartl. Extremophiles, 16(1), 95-103.

20. Wang, T., Yang, S., Petrenko, V. A., \& Torchilin, V. P. (2010). Cytoplasmic delivery of liposomes into MCF-7 breast cancer cells mediated by cell-specific phage fusion coat protein. Molecular pharmaceutics, 7(4), 1149-1158.

\section{Tables}

Table 1. Substituents (R) of 4-(2-Keto-1-benzimidazolinyl)piperidine (I) derivatives 


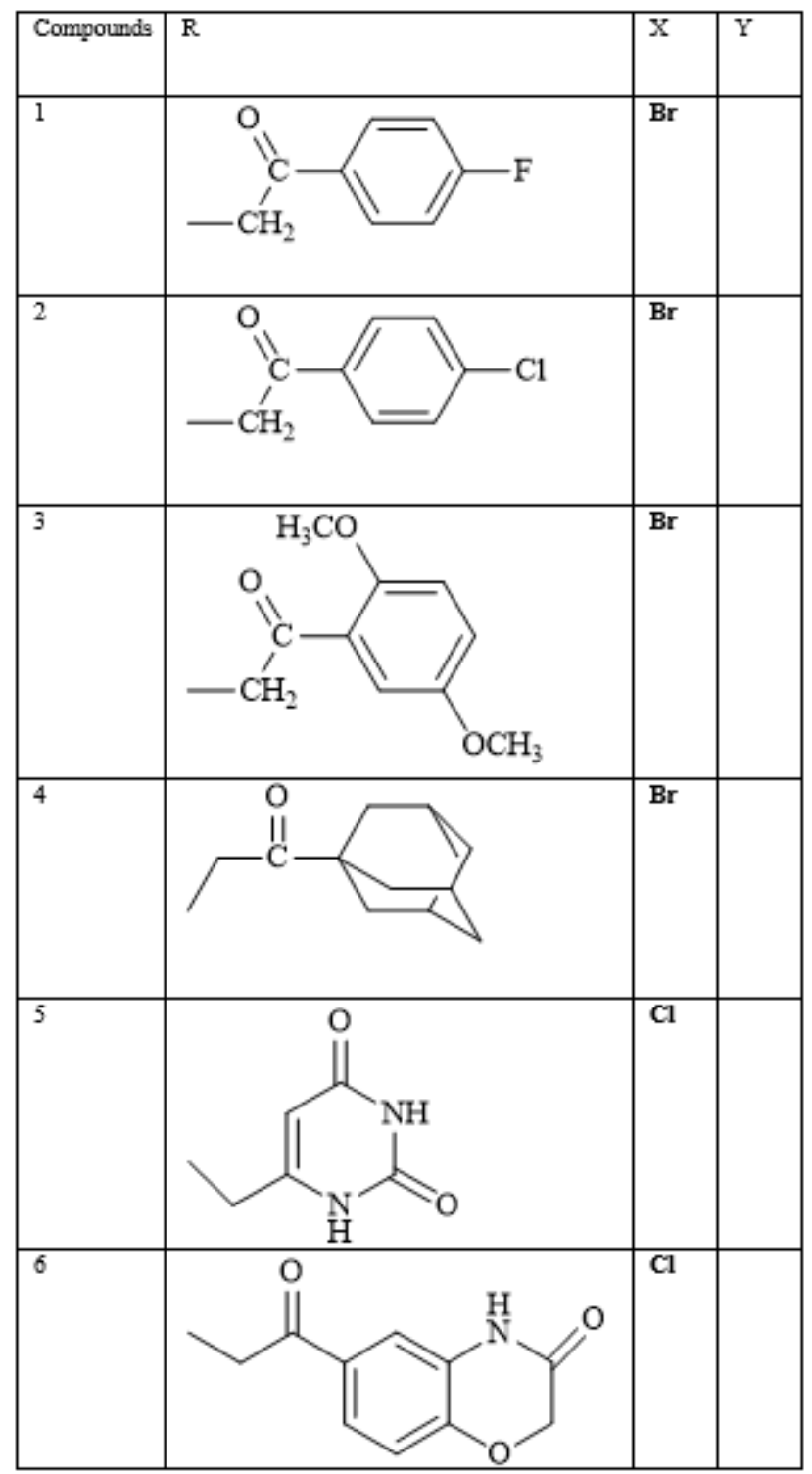

Table 2. Substituents ( $R$ ) of 5-Chloro-1-(4-piperidyl)-2-benzimidazolinone (II) derivatives 


\begin{tabular}{|l|l|l|}
\hline Compounds & & $\mathrm{X}$ \\
\hline 7 & & $\mathrm{~B}$ \\
\hline 8 & & \\
\hline 9 & & \\
\hline 10 & & \\
\hline
\end{tabular}

Table 3. Fluorescence (R.F.U.) and cytotoxicity (\%) of the human cervical cancer cells after $24 \mathrm{~h}$ exposure of the synthesized compounds (1-11) at 100 uM concentration. 


\begin{tabular}{|c|c|c|}
\hline Compound $(100 \mu \mathrm{M})$ & Avg Fluorescence (R.F.U.) & Cytotoxicity (\%) \\
\hline 1 & 3631.2 & -10.1 \\
\hline 2 & 3929.8 & -19.2 \\
\hline 3 & 1838.9 & 44.2 \\
\hline 4 & 1296.0 & 60.7 \\
\hline 5 & 2353.0 & 28.6 \\
\hline 6 & 3442.9 & -4.4 \\
\hline 7 & 2067.7 & 37.3 \\
\hline 8 & 3929.8 & -19.2 \\
\hline 9 & 1286.6 & 61.0 \\
\hline 10 & 725.3 & 78.0 \\
\hline 11 & 2337.3 & 29.1 \\
\hline C & 3929.8 & -19.2 \\
\hline
\end{tabular}

R.F.U. = Relative Fluorescence Unit; C = Control.

Table 4. Fluorescence (RFU) of the compounds 4, 9 and 10 at 10,50 and 100 uM concentration on human cervical cancer cells after $24 \mathrm{~h}$ exposure.

\begin{tabular}{|llll|}
\hline \multicolumn{4}{|l|}{ Fluorescence (RFU) } \\
\begin{tabular}{|l} 
Compound \\
Conc. $(\mu \mathrm{M})$
\end{tabular} & & 9 & 10 \\
\hline 10 & 10690.994 & 6962.775 & 2729.123 \\
\hline $\mathbf{5 0}$ & 6390.205 & 5094.387 & 2033.234 \\
\hline $\mathbf{1 0 0}$ & 1413.752 & 1410.242 & 1354.971 \\
\hline $\mathrm{C}$ & 8879.760 & 8879.760 & 8879.760 \\
\hline
\end{tabular}

R.F.U. = Relative Fluorescence Unit; C = Control.

Table 5. The cytotoxicity (\%) of the compounds 4, 9 and 10 at 10, 50 and 100 uM concentration on human cervical cancer cells after $24 \mathrm{~h}$ exposure. 


\begin{tabular}{|llll|}
\hline \multicolumn{3}{|l|}{ Cytotoxicity } \\
\hline $\begin{array}{l}\text { Compound } \\
\text { Conc. }(\mu \mathrm{M})\end{array}$ & & 9 & 10 \\
\hline 10 & -20.397 & 21.588 & 69.266 \\
\hline 50 & 28.036 & 42.629 & 77.103 \\
\hline 100 & 84.079 & 84.118 & 84.741 \\
\hline C & 0.000 & 0.000 & 0.000 \\
\hline
\end{tabular}

C = Control

Table 6. In-vitro leishmanicidal activity of benzimidazolinyl piperidine derivatives.

\begin{tabular}{|ll|}
\hline Compounds & $\mathrm{IC}_{50}(\mu \mathrm{g} / \mathrm{mL}) \pm$ SEM $^{\mathrm{a}}$ \\
\hline 1 & $43.92 \pm 1.511$ \\
\hline 2 & $75.46 \pm 0.465$ \\
3 & $27.41 \pm 1.52$ \\
\hline 4 & $7.06 \pm 0.17$ \\
\hline 5 & $\mathrm{NA}$ \\
\hline 6 & $\mathrm{NA}$ \\
\hline 7 & $46.986 \pm 0.243$ \\
\hline 8 & $72.4 \pm 1.74$ \\
\hline 9 & $84.6 \pm 0.6$ \\
\hline 10 & $23.24 \pm 0.035$ \\
\hline 11 & $\mathrm{NA}$ \\
\hline I & $\mathrm{NA}$ \\
\hline II & $51.68 \pm 0.02$ \\
\hline Amphotericin $\mathrm{B}^{\mathrm{b}}$ (st) & $0.29 \pm 0.05$ \\
\hline Pentamidine ${ }^{\text {(st })}$ & $5.09 \pm 0.09$ \\
\hline
\end{tabular}


$\mathrm{SEM}^{\mathrm{a}}=$ standard error of mean;NA ${ }^{\mathrm{b}}=$ Not active; amphotericin $\mathrm{B}^{(\mathrm{st})}$ and pentamidine $\mathrm{s}^{(\mathrm{st})}=$ standard drugs for leishmanicidal activity.

\section{Scheme}

Scheme $1 \& 2$ Is Available In Supplemental Files Section.

\section{Supplementary Files}

This is a list of supplementary files associated with this preprint. Click to download.

- 1.png

- 2.png 